\title{
Acute Myocardial Infarction in Young Adults: Study of Risk factors, Angiographic Features and Clinical Outcome
}

\author{
Rikesh Tamrakar*1, Yadav Deo Bhatt ${ }^{2}$, Subodh Kansakar $^{2}$, Mahesh Bhattarai ${ }^{3}$, \\ Kunal Bikram Shaha², Eanstara Tuladhar ${ }^{3}$ \\ ${ }^{1}$ National Academy of Medical Sciences, Kathmandu ,Nepal \\ ${ }^{2}$ Shahid Gangalal National Heart Center, Kathmandu,Nepal \\ ${ }^{3}$ Institue of Medicine,Tribhuwan University , Kathmandu, Nepal
}

\begin{abstract}
Citation
Tamrakar R, Bhatt YD, Kansakar S, et al. Acute Myocardial Infarction in Young Adults: Study of Risk factors, Angiographic Features and Clinical Outcome. Nepalese Heart Journal 2013;10(1):12-16
\end{abstract}

\section{Keywords}

acute myocardial infarction; coronary angiography; coronary artery disease; risk factors.

\begin{abstract}
Background and aims: Acute myocardial infarction below 45 years of age constitutes a specific subset of population having different risk factors and clinical features as compared to older patients. Pattern of coronary artery involvement and clinical outcome also varies suggesting different underlying pathophysiology. Better understanding this specific problem will lead to further improvement in management.

Methods: One hundred and fifteen consecutive patients diagnosed as acute myocardial infarction and were below 45 years over the period of one year were enrolled in the study. Clinical parameters, risk factors, angiographic patterns were analysed. Patients were assessed daily during hospital stay to study in-hospital outcome and complications.

Results: Out of total patients studied, $64.3 \%$ were smokers, $27.8 \%$ were hypertensive, $15.65 \%$ were diabetic and $9.6 \%$ had history of dyslipidemia. There was history of recreational drug abuse in $4.3 \%$ of patients. Most patients present as ST elevation myocardial infarction (87\%). Majority of patients were in Killip class I (69.5\%) at presentation in emergency. Single vessel disease (58.3\%) was the most common finding in coronary angiography and $7.6 \%$ patients have normal or non obstructive coronary lesions. Complications include arrhythmia (6\%), cardiogenic shock (5.2\%) and mortality (1.7\%).

Conclusions: In young acute myocardial infarction patients, smoking is the single most important modifiable risk factor. Other conventional risk factors are less strongly associated than older patients. Young patients tend to have less extensive coronary artery lesions. In few patients, presence of normal or non obstructive coronary lesions would suggest possibility of different mechanism of myocardial necrosis. Overall, these patients had favourable outcome and better prognosis.
\end{abstract}

\section{INTRODUCTION}

Coronary Artery Disease (CAD) is the major cause of morbidity and mortality burden in the world. Young patients with CAD are specific subset of population requiring attention. The cut off age of

\section{*Corresponding Author:}

Rikesh Tamrakar

National Academy of Medical Sciences

Mahaboudha, Kathmandu,Nepal

Email:riktam77@gmail.com 
45 has been used in most studies to define young patients with CAD and myocardial infarction. ${ }^{1}$ Although uncommon entity, it constitutes an important problem for the patient and the treating physician because of the devastating effect of this disease on the more active lifestyle of young adults. In addition, these patients have different risk factor profiles, and prognosis than older patients. $^{2,3}$ A variety of other possible contributing factors that include substance abuse, coronaryartery anomalies, hypercoagulablestate, oral contraceptive use in young women have been implicated for the pathogenesis of myocardial infarction. ${ }^{4,5}$ The clinical presentation is also different from that of older patients. In majority of cases, a sudden myocardial infarction or unstable angina is the first manifestation of CAD. 6,7. Angiographic studies also showed major differences with higher incidence of normal coronary arteries, mild luminal irregularities, and single vessel coronary artery disease. ${ }^{8}$

Cardiovascular risk factors for ischemic heart disease and acute myocardial infarction are on the rise in Nepalese population. Recent observation has shown the significant rise of patients in younger age. But there is currently very little data regarding studies of young acute myocardial infarction (AMI) in our population. The purpose of this study is to collect data of young patients presenting with AMI so that it would be helpful in future for better management in this particular group of patients.

\section{METHODS}

This Prospective observational study was carried out in Shahid Gangalal National Heart Center (SGNHC) over period of one year from June 2012 to June 2013. Consecutive patients below 45 years and diagnosed as acute myocardial infarction according to current guidelines, attending SGNHC were included in the study.

Patients were diagnosed with one of the following 1) ST segment elevation MI (STEMI), 2) Non-ST segment elevation MI (NSTEMI). STEMI was diagnosed by having elevated biochemical markers of myocardial necrosis and ECG changes demonstrating either: 1) ST segment elevation $>1$ $\mathrm{mm}$ in two consecutive leads or 2) new or presumed new left bundle branch block along with ischemic symptoms. NSTEMI was determined by elevated biochemical markers of myocardial necrosis and either ischemic symptoms compatible with ACS or ST-segment depression or T wave abnormalities.

A brief questionnaire that included the history of diabetes, hypertension, stroke and family history of ischemic heart disease were recorded. It also included the demographic data, smoking habit, and physical activity. Fasting blood glucose, renal function and lipids were measured within 24 hours of admission of the case subjects. Patients below 18 years and above 45 years were excluded from the study. Similarly patients having prior cardiac conditions that could affect outcome like valvular heart disease, cardiomyopathy, and previous left bundle branch block were excluded. Patients with diagnosed acute or chronic liver disease, renal impairment or having secondary conditions that could precipitate angina (anemia, arrhythmias, fever) were also excluded from the study.

Diabetes was defined as having a history of diabetes diagnosed and/or treated with medication and/or diet or fasting blood glucose $7.1 \mathrm{mmol} / 1$ or greater. Hypertension was defined as having a history of hypertension diagnosed and/or treated with medication, diet, blood pressure greater than $140 \mathrm{mmHg}$ systolic or $90 \mathrm{mmHg}$ diastolic on at least two occasions, or as receiving any antihypertensive drug. Dyslipidemia was defined as history of dyslipidemia diagnosed and/or treated by a physician or total cholesterol greater than $5.18 \mathrm{mmol} / \mathrm{l}$, low-density lipoprotein greater than or equal to $3.37 \mathrm{mmol} / \mathrm{l}$, or high-density lipoprotein $<1.04 \mathrm{mmol} / \mathrm{l}$. High triglyceride (TAG) level was defined as level more than $2.3 \mathrm{mmol} / \mathrm{L}$. Current smoker was defined as a person who reported smoking at least 100 cigarettes in their lifetime and who, at the time of survey, smoked either every day or some days. Obesity was defined as body mass index greater than $25 \mathrm{~kg} / \mathrm{m}^{2}$

All patients were managed in coronary care unit (CCU) according to hospital protocol and current guidelines. Besides, dual antiplatelets, statins; patients also receive angiotensin converting enzyme or angiotensin II receptor blocker and beta blocker as indicated. The interventions done in the patients and their outcome were recorded. Patients were followed every day during the hospital stay to record progress and complication.

Descriptive statistics were used to describe the data. For categorical variables, frequencies and percentages were reported. Differences between groups were analyzed using Pearson's $\chi^{2}$ tests (or Fisher's exact tests for cells $<5$ ). For continuous variables, means and standard deviations were presented and analyses were conducted using Student's $t$-test. A level of significance was set at the 0.05 level. Statistical analyses were conducted using SPSS version 17.0

\section{RESULTS}

Out of 115 patients included in the study, 85 (74 $\%)$ were males and $30(26 \%)$ were females. Only five patients were below 30 years of age, rest fall 
between 30 to 45 years of age.

Tobacco consumption emerged as single most important risk factor for AMI in young patients. Of total patients, $64.3 \%$ of the patients were current smoker, $10 \%$ were past smoker. Five patients $(4.3 \%)$ had history of recreation drug abuse. Surprisingly $4.3 \%$ of patients had been taking psychotrophic medication. Three women were using oral contraceptive pills.

Majority of the patients did not have conventional risk factors like diabetes, hypertension, and dyslipidemia. However, there was trend to have higher level of TAG level in our study population; $68 \%$ patients had TAG level more than cutoff value of $2.3 \mathrm{mmol} / \mathrm{l}$. Just five patients would fulfil criteria for obesity. Prevalence of each modifiable risk factor is given in table 1 .

Only $19.1 \%$ of the patients present within three hours of onset of symptoms, $20 \%$ between three to six hours, $23.5 \%$ between 6 to 12 hours, $37.4 \%$ present beyond 12 hours. At presentation, 69.5\%, $20.1 \%, 5.2 \%$ and $5.2 \%$ of patients were in Killip class I, II, III and IV respectively.

Most of the patients $(87 \%)$ presented as STEMI while only $13 \%$ were diagnosed as non ST elevation myocardial infarction. Clinical diagnosis of myocardial infarction is given in table 2 .

Regarding management, $37 \%$ patients presented late for revascularization and were managed conservatively and $31 \%$ patients were thrombolysed with streptokinase. Other $32 \%$ patients underwent primary percutaneous intervention (PCI). In primary PCI group, stents were deployed in $94.2 \%$ patients. No intervention or only balloon angioplasty / thrombosuction were done in $5.2 \%$ of patients. Rescue PCI after failed thrombolysis was done in $5.2 \%$ patients.

Coronary angiography (CAG) was performed in total of $56.5 \%$ patients during the hospital stay. Single vessel disease was most common finding followed by double vessel disease and triple vessel disease. Left anterior descending artery was most commonly involved. $6.9 \%$ patients had non critical $\mathrm{CAD}$ or normal coronary angiogram. CAG finding is shown in table 3 .

Regarding the complications, 5.2\% patients developed cardiogenic shock requiring inotropes, $6 \%$ had significant arrhythmias requiring intervention. There were only two deaths (1.7\%) during hospital, both diagnosed as extensive anterior wall MI on admission. Mortality was less as compared to overall $7 \%$ mortality from MI from hospital registry.

\section{DISCUSSION}

In this study of young patients with AMI, we find some important differences as compared to older patients. So, even though they form small proportion of total AMI cases; it is an important group to examine for the purpose of risk factor modification and secondary prevention.

As expected, there is male predominance. We find smoking as a single most risk factor. Study by Zimmerman et $\mathrm{al}^{9}$ found that among AMI patients $<40$ years old $73 \%$ to $90 \%$ reported a history of smoking. Many other studies have also found high rates of tobacco use among young patients who have AMI, with percentages ranging from $70 \%$ to $>90 \%{ }^{10-12}$ Smoking is known to cause increased fibrinogen concentrations and platelet aggregability, along with impaired fibrinolytic activity, decreased coronary flow reserve, and increased vasospasm. ${ }^{13-15}$ Recurrent exposure to cigarettes with subsequent catecholamine surges damage endothelial cells, leading to endothelial dysfunction and injury of the vascular intima. Autopsy studies in young adults have showed that the extent of fatty-streak lesions in the coronary arteries of young adults was higher in smokers than in non-smokers. ${ }^{16}$ All these evidence point out smoking as most important modifiable risk factor in young adults.

We find the conventional risk factors like hypertension, diabetes, dyslipidemia or obesity was less prevalent as compared to older patients. However, there was higher TAG and low HDL level. Similar finding has also been reported in other studies of AMI patients under age 45 years. ${ }^{17} \mathrm{~A}$ meta-analysis of 16 prospective epidemiologic studies suggests that an elevated TAG concentration is an independent risk factor for coronary artery disease. ${ }^{18}$ So, we may infer that high TAG, which may be the primary lipid abnormality with or without low HDL levels and normal/low LDL levels is a matter of concern in young adults.

CAG though not done in all patients in our study revealed some important findings. We find young patients tend to have less extensive atheromatous lesions. The contribution of prothrombotic and inflammatory indices may be particularly implicated in the initiation and development of such atherosclerotic lesions. ${ }^{19}$ Moreover, the predominance of angiographic single vessel disease in these young patients as in our study primarily suggest that premature myocardial infarction probably result from a rapid progressive event, such as thrombosis or plaque rupture, rather than a gradually evolving process, such as atherosclerosis, and thus substantiate the need for an intense and aggressive approach directed towards primary and secondary preventions of premature cardiovascular disease. ${ }^{20}$ Myocardial Infarction with Normal Coronary Arteries (MINCA) is an important subgroup of myocardial infarction in young patients with a frequency of at least $3-4 \%$ of all myocardial 
infarctions. ${ }^{21}$ In the present study also, $6.9 \%$ of coronary angiography were normal or had non critical CAD. The proposed mechanisms for MINCA include coronary vasospasm, coronary thrombosis in situ or embolization from a distal source with spontaneous lysis, drug abuse, viral myocarditis, aortic dissection, hypercoagulable states, autoimmune vasculitis and carbon monoxide poisoning. ${ }^{21}$ Though this study was not designed to find out underlying pathophysiology of AMI, higher prevalence of drug abuse and other medication may indicate similar mechanism for AMI.

Finally, we find younger patients were significantly more likely to be in Killip class I, and have better in hospital prognosis. Various other studies showed the similar inhospital mortality rate among young MI patients of the approximately $1 \%$ to $6 \%$, compared with $8 \%$ to $22 \%$ in older patients. ${ }^{22}$ Although younger patients haven favorable short term prognosis, the personal and social burden is substantial. Thus, preventive measures must be addressed early to decrease long term morbidity and mortality from coronary artery disease.

Our study has certain limitations. First it is an observational study so certain confounding variables could have played role. Not all patents underwent angiographic procedure, which could have influenced the results. We only analyze the patients who reached the hospital so it might not be a true representative of the population. Utilization of thrombolytic therapy could have potentially affected our results as far as the higher incidence of normal coronary arteries is concerned.

\section{CONCLUSIONS}

Young patients diagnosed with AMI have some important differences that should be appreciated. The most important modifiable risk factor is tobacco smoking. Other conventional risk factors are not strongly associated as in older patients. They are more likely to have less extensive coronary artery disease. A careful search for other contributing factors is warranted as progressive atherosclerosis may not always be the underlying pathophysiology. If timely managed, young patients with AMI have favourable inhospital prognosis.

\section{Table 1: Risk factors among the patients}

\begin{tabular}{lcc} 
Risk Factors & \% & Number (115) \\
Smoking & $64.3 \%$ & 74 \\
Hypertension & $27.8 \%$ & 32 \\
Diabetes & $15.6 \%$ & 18 \\
Dyslipidemia & $9.6 \%$ & 11 \\
Family history & $9.6 \%$ & 11 \\
Obesity & $4.3 \%$ & 5 \\
\hline
\end{tabular}

Table 2: Clinical diagnosis of the patients

\begin{tabular}{lcc|}
\hline \multicolumn{1}{c}{ Clinical diagnosis } & $\%$ & $\begin{array}{c}\text { Number } \\
(115)\end{array}$ \\
\hline Anterior wall & $36.5 \%$ & 42 \\
Extensive Anterior wall & $14 \%$ & 16 \\
Inferior+ right ventricular & $7.8 \%$ & 9 \\
Inferior / inf posterior wall & $26 \%$ & 30 \\
Lateral wall & $2.6 \%$ & 3 \\
NSTEMI & $13 \%$ & 15 \\
\hline
\end{tabular}

\section{Table 3: Coronary angiographic findings}

\begin{tabular}{lcc}
\hline $\begin{array}{c}\text { Coronary angiographic } \\
\text { finding }\end{array}$ & \% & $\begin{array}{c}\text { Number } \\
(\mathbf{6 5 )}\end{array}$ \\
\hline Single Vessel Disease & $53.8 \%$ & 35 \\
LAD & $27.6 \%$ & 18 \\
LCX & $10.7 \%$ & 7 \\
RCA & $15.3 \%$ & 10 \\
Double Vessel Disease & $36.9 \%$ & 24 \\
LAD + LCX & $18.4 \%$ & 12 \\
LAD + RCA & $10.7 \%$ & 7 \\
LCX + RCA & $7.6 \%$ & 5 \\
Triple Vessel Disease & $6.1 \%$ & 4 \\
Normal/ Non critical CAD & $7.6 \%$ & 5 \\
\hline
\end{tabular}

\section{REFERENCES}

1. $\mathrm{M}$ Egred, $\mathrm{G}$ Viswanathan, $\mathrm{G} \mathrm{K}$ Davis. Myocardial infarction in young adults. Postgrad Med J. 2005;81:741-745.

2. Chouhan L, Hajar HA, Pomposiello JC. Comparison of thrombolytic therapy for acute myocardial infarction in patients aged $<35$ and $>55$ years. Am J Cardiol. 1993;15;71(2):157-9.

3. Hoit BD, Gilpin EA, Henning H, Maisel
AA, Dittrich H, Carlisle J, et al. Myocardial infarction in young patients: an analysis by age subsets. Circulation. 1986;74(4):712-21.

4. Al-Koubaisy OK, Mehdi RS, Arem FD, Ahmed IT. Cine angiographic findings in young Iraqi men with first acute myocardial infarction. Cathet Cardiovasc diagn. 1990;19:87-90.

5. Rosenberg, L, Kaufman, DW, Helmrich, SP, Miller DR, Stolley PD, Shapiro S. Myocardial 
infarction and cigarette smoking in women younger than 50 years of age. JAMA. 1985; 253:2965-9.

6. Ruotolo G, Bavenholm P, Brismar K, Eféndic S, Ericsson CG, de Faire U, et al. Serum insulin-like growth factor-I level is independently associated with coronary artery disease progression in young male survivors of myocardial infarction: beneficial effects of bezafibrate treatment. J Am Coll Cardiol. 2000; 35:647-54.

7. Fournier, JA, Sanchez, A, Quero J, Fernández-Cortacero JA, González-Barrero A. et al. Myocardial infarction in men aged 40 years or less: a prospective clinical-angiographic study. Clin Cardiol. 1996;19:631-6.

8. Klein, LW, Agarwal, JB, Herlich, MB, Leary TM, Helfant RH. Prognosis of symptomatic coronary artery disease in young adults aged 40 years or less. Am J Cardiol. 1987; 60:1269-72

9. Zimmerman FH, Cameron A , Fisher LD, Grace NG. Myocardial infarction in young patients: angiographic characterization, risk factors and prognosis (Coronary Artery Surgery Registry). J Am Coll Cardiol . 1995;26:654661.

10. Choudhury L, Marsh JD. Myocardial infarction in young patients. Am J Med. 1999;107:254-61.

11. Hong MK, Cho SY, Hong BK, Chang KJ, Mo-Chung I, Hyoung-Lee $\mathrm{M}$, et al. Acute myocardial infarction in young adults. Yonsei Med J. 1994;35(2):184-9.

12. Adhikari CM, Rajbhandari R, Limbu YR, Malla R, Sharma R, Rauniyar B, et al. A study on major cardiovascular risk factors in acute coronary syndrome (ACS) patient 40 years and below admitted in CCU of Shahid Gangalal National Heart Center. Nepalese Heart Journal. 2010;7(1):20-24.

13. Panduranga P., Sulaiman K., Al-Zakwani I., Abdelrahman S. Acute coronary syndrome in young adults from Oman: results from the Gulf registry of acute coronary events. Heart Views. 2010;11(3):93-98.
14. Lang NN, Gudmundsdóttir IJ, Boon NA, Ludlam CA, Fox KA, Newby DE. Marked impairment of protease-activated receptor type 1-mediated vasodilatation and fibrinolysis in cigarette smokers: Smoking, thrombin, and vascular responses in vivo. J Am Coll Cardiol. 2008;52:33-9.

15. Morita K, Tsukamoto $T$, Naya $M$, Noriyasu K, Inubushi M, Shiga $\mathrm{T}$, et al. Smoking cessation normalizes coronary endothelial vasomotor response assessed with 15O-water and PET in healthy young smokers. J Nucl Med. 2006;47:1914-20.

16. Berenson GS, Srinivasan SR, Bao W, Newman WP 3rd, Tracy RE, Wattigney WA. Association between multiple cardiovascular risk factors and atherosclerosis in children and young adults. The Bogalusa Heart Study. N Engl J Med 1998;338:1650-6.

17. Chen L, Chester M, Kaski JC. Clinical factors and angiographic features associated with premature coronary artery disease. Chest 1995; 108:364 -369.

18. Farmer JA, Gotto AM, Jr. Dyslipidemia and other risk factors for coronary artery disease. In: Braunwald E, ed. Heart Disease: A Textbook of Cardiovascular Medicine. Philadelphia: Saunders. 1997:1126-1160.

19. Alkhiary W. Premature Myocardial Infarction: An updated Overview. Webmed Central Cardiology. 2011;2(12):WMC002597

20. Bajaj S, Shamoon F, Gupta N, Parikh R, Parikh $\mathrm{N}$, Debari VA, et al. Acute ST-segment elevation myocardial infarction in young adults: Who is at risk? Coron Artery Dis. 2011;22:238-44.

21. Agewall S, Eurenius L, Hofman-Bang C, Malmqvist K, Frick M, Jernberg $\mathrm{T}$, Tornvall P Myocardial infarction with angiographically normal coronary arteries. Atherosclerosis. 2011. 219:10-4.

22. Rumboldt Z, Rumboldt M, Pesenti S, Polic S, Miric D. Peculiarities of myocardial infarction at young age in Southern Croatia. Cardiologia. 1995;40:407- 411 . 